Sociologie et sociétés

SOCIOLOGIE

ETSOCIÉTÉS

\title{
Spectacles spécifiques
}

Critique, assomption et régression du spectaculaire dans le système de l'art contemporain

Specific Spectacles
Critique, Assumption and Regression of the Spectacular in the Contemporary Art System

\section{Louis Jacob}

Volume 37, numéro 1, printemps 2005

Le spectacle des villes

The Spectacle of Cities

URI : https://id.erudit.org/iderudit/012280ar

DOI : https://doi.org/10.7202/012280ar

Aller au sommaire du numéro

\section{Éditeur(s)}

Les Presses de l'Université de Montréal

\section{ISSN}

0038-030X (imprimé)

1492-1375 (numérique)

Découvrir la revue

\section{Citer cet article}

Jacob, L. (2005). Spectacles spécifiques : critique, assomption et régression du spectaculaire dans le système de l'art contemporain. Sociologie et sociétés, 37(1), 125-150. https://doi.org/10.7202/012280ar

\section{Résumé de l'article}

L'article attire l'attention sur un ensemble de pratiques artistiques qui relèvent de l'« intervention », de la " performance » et de l'« installation » et qui entendent déconstruire le spectacle des villes. Les interventions artistiques en milieu urbain remettent en question les catégories usuelles de l'art et du spectateur, mais aussi celles de l'action culturelle, de l'interaction et de la communauté. Partant de l'hypothèse générale que les " oeuvres d'art » - qui ne sont pas nécessairement des " objets " - sont des configurations signifiantes, on examine les discours critiques et esthétiques qui concourent à la définition du genre, puis les éléments clés du champ de l'intervention artistique : la revitalisation urbaine, les politiques culturelles, les réseaux artistiques, les nouveaux espaces publics et les formes de participation. L'article vise à démontrer que les artistes qui pratiquent l'intervention urbaine doivent directement faire face à la difficulté de penser le nouvel espace public et de le concrétiser.
Tous droits réservés @ Les Presses de l'Université de Montréal, 2005
Ce document est protégé par la loi sur le droit d'auteur. L'utilisation des services d'Érudit (y compris la reproduction) est assujettie à sa politique d'utilisation que vous pouvez consulter en ligne.

https://apropos.erudit.org/fr/usagers/politique-dutilisation/ 


\title{
Spectacles spécifiques:
}

\author{
critique, assomption et régression \\ du spectaculaire dans le système de l'art contemporain
}

\section{LOUIS JACOB}

Département de sociologie

Université du Québec à Montréal

C. P. 8888 , Succursale Centre-ville

Montréal (Québec) Canada $\mathrm{H}_{3} \mathrm{C}_{3} \mathrm{P} 8$

Courriel: jacob.louis@uqam.ca

\section{$\mathrm{L}$} E TITRE « SPECTACleS SPÉCIFIQUeS » fait référence à un article phare de Donald Judd, «Specific Objects», paru dans Arts Yearbook en 1965. L'article, repris aujourd'hui dans plusieurs anthologies, annonçait en quelque sorte l'offensive minimaliste dans le monde de l'art. Judd en avait contre les multiples manifestations de l'« illusionnisme » en peinture et en sculpture, jusque dans les œuvres de l'avant-garde. Il revendiquait le caractère autotélique ou autopoiétique de l'œuvre d'art, un caractère désormais parfaitement admis (et contesté de toutes parts, cela va de soi) qui se traduit dans le contexte actuel par un paradoxe particulièrement fécond: la possibilité d'une ouverture radicale de l'art à tout ce qui n'est pas de l'art, et la nécessité pour l'art de redéfinir sans cesse son autonomie dans un environnement complexe avec lequel il entre de plus en plus étroitement en relation. L'art peut être de l'art et déjouer toutes les attentes d'ordre esthétique, c'est-à-dire sans donner l'illusion d'art.

L'objet de ce texte est double. Je voudrais d'abord attirer l'attention sur un ensemble de pratiques artistiques très différentes de celles qu'envisageait Judd, des pratiques artistiques qui relèvent de l' «intervention ", de la "performance» et de l'« installation », et qui revendiquent pour elles une spécificité tout aussi radicale, à la fois par rapport au concept d'art et au système dans lequel il s'inscrit, et par rapport au mode spectaculaire qui se manifeste partout dans la société contemporaine. J'aimerais ensuite démontrer que les artistes 
qui pratiquent l'intervention urbaine doivent faire face à la difficulté de penser le nouvel espace public et de le concrétiser. Je pars de l'hypothèse générale que les « œuvres d'art»qui ne sont pas nécessairement des « objets»-sont des configurations signifiantes à partir desquelles il est possible d'exercer un jugement rationnel, et qu'elles ne relèvent pas que de l'expérience esthétique : elles ont aussi des effets d'ordre normatif, cognitif et pragmatique repérables dans un contexte donné.

Les œuvres dont il sera question se distinguent en ce qu'elles entendent déconstruire le spectacle des villes. Elles remettent ainsi en question les catégories usuelles de l'art et du spectateur, mais aussi celles de l'action culturelle, de l'interaction et de la communauté. Ces œuvres sont dites furtives (Loubier), inorganiques ou contextuelles (Ardenne), relationnelles (Bourriaud), sociologiques (Fisher). Ce sont des formes d'actions urbaines (Augoyard) dites aussi participatives ou citoyennes (Doyon/Demers). Elles se conçoivent elles-mêmes comme «stratégie», "manœuvre» ou « entreprise» (Sioui Durand; Richard).

Je ne proposerai que de manière très incidente des analyses d'interventions artistiques particulières (un seul cas sera analysé plus longuement), mon propos étant bien davantage de cerner une situation générale. De même, je ne me penche pas directement sur la notion d' «art public». Si on admet que la notion d'art public désigne toute forme d'art qui occupe, de façon transitoire, plus ou moins durable ou permanente, l'espace public (et on verra que c'est précisément la définition de cet espace qui est en jeu), alors certaines «interventions artistiques urbaines» font bien partie de cette grande famille de l'art public, même si elles cohabitent avec des œuvres qui, de prime abord, paraissent parfaitement étrangères, par exemple sur le plan de l'intention: répondre à une commande ou une demande sociale, s'emparer d'un lieu, créer un espace d'expérimentation artistique, etc.

L'identification des genres et la construction sociale des valeurs propres au monde de l'art ne constituent pas une question interdite en sociologie, et elle n'est certainement pas triviale. Au contraire, comme le soulignait Vera Zolberg (1990, p. 193-195), ces distinctions ou ces frontières aujourd'hui particulièrement mouvantes entre les types de pratiques et entre les normes qui accompagnent le jugement esthétique ainsi que les comportements culturels en général sont devenues une question centrale qui permet de comprendre non seulement le système de l'art mais aussi certains des enjeux essentiels de la société contemporaine. Howard S. Becker $(1982,1983)$ a attiré notre attention sur l'aspect collectif ou coopératif des activités artistiques, depuis la création jusqu'à la réception des œuvres, et sur l'interaction des nombreux agents qui participent, avec les artistes eux-mêmes, à la définition des conventions, des genres, des réputations, dans des «mondes de l'art » différenciés. Chacun à sa manière, Bourdieu $(1979,1992)$, DiMaggio $(1987,1991)$ et Luhmann (2000) ont eux aussi abondamment étudié la question des frontières normatives de l'art, mais ils n'abordent que très indirectement l'autre question concomitante, celle du contenu artistique ou culturel des œuvres et leur signification sociale.

La sociologie de l'art a donc pris l'habitude de mettre à plat l'ensemble des médiations et des relations qui définissent l'œuvre, l'artiste, l'institution. Ce déploiement des dimensions à la fois sémiotiques et matérielles du système de l'art laisse peu de place pour discuter de la contribution de la sociologie de l'art à la théorie générale de la société. On est pourtant en droit de s'attendre à ce genre de discussion lorsqu'on s'interroge sur le 
spectacle des villes et les formes spectaculaires de la ville. C'est ce qui va être tenté ici, en partant du cas des interventions artistiques en milieu urbain, plus précisément à Montréal, considérées dans leurs rapports avec le spectaculaire. Le texte se situe donc aussi au confluent de courants, par ailleurs assez disparates, qui militent pour le renouveau de la sociologie de la culture ou qui font valoir la centralité de la culture au sein de la sociologie générale (Alexander et Smith, 1998 ; Côté, 1998 ; Hays, 2000 ; Lamont, $2000^{1}$ ).

\section{LA DÉFINITION D'UN GENRE}

Esquissons pour commencer les frontières du genre, à travers notamment les diverses traditions critiques et esthétiques qui sont sollicitées dans le monde de l'art lui-même. Le jeu de la taxinomie qui entoure la définition de l'« intervention artistique» est tout sauf superficiel ou accessoire. Dans les cercles restreints de la recherche et de la création en art contemporain, c'est même un enjeu déterminant. Il s'agit bien entendu d'une prise de position dans le champ artistique (Bourdieu, 1992 ; Heinich, 1992), mais c'est aussi, et peut-être surtout, le sens de l'activité et son inscription dans l'espace social qui en dépendent. Il faut à ce propos souligner que l'activité théorique, critique ou discursive n'émane pas seulement des médiateurs ou des institutions, mais des artistes eux-mêmes et des citoyens qui participent, qui entrent en relation, qui créent des situations et des relations sociales inédites. On peut à cet égard parler d'un « dédoublement» et d'une « réinscription » de la relation esthétique, pour le dire comme Luhmann.

L'appellation «interventions artistiques en milieu urbain », bien qu'elle ait largement cours dans le monde de l'art depuis la fin des années 1970, recouvre en fait une grande variété d'enjeux formels, stylistiques et pragmatiques. La sociologie de l'art rend compte des stratégies de valorisation, de marquage, de labellisation, de tout le processus de construction sociale sous-jacent au type ou au genre, à l'œuvre et son contexte de signification. Par ailleurs, ces interventions artistiques, et le label «urbain» qui les qualifie, renvoient aussi à un imaginaire de la ville, une configuration particulière d'images sensibles ou mentales, c'est-à-dire une expérience subjective de la ville, que l'on peut mesurer, associer ou opposer aux autres discours sociaux. La perspective générale qui est esquissée ici devrait tenir compte de la dimension de l'imaginaire, où l'œuvre apparaît comme problématisation de l'expression, de la communication, de la construction des identités et des volontés.

1. Je m’appuie sur des discussions auxquelles j’ai participé depuis quelques années au sein du Réseau interuniversitaire de recherche sur les arts, la culture et la société, ainsi que sur celles qui ont entouré la conception et la mise en place d'une base de données sur les œuvres d'art public permanentes et éphémères, au Centre d'information Artexte auquel j'ai été associé en tant que chargé de projet. Outre les discussions régulières avec les artistes, les chercheurs et les spécialistes, j'ai aussi complété quatre entretiens en profondeur avec des professionnels du monde de l'art sur ces questions, entre mai et juillet 2003. Je reprends également quelques extraits d'un rapport rédigé à l'attention de Daniel Latouche, dans le cadre d'un travail de recherche en collaboration, et dont il a tiré une présentation intitulée «Les arts et la ville», lors du Congrès du Comité de recherche en sociologie de l'art de l'AIs, «The Culture Society: A New Place for the Arts in the Twenty-First Century», Barcelone, 6-8 juillet 2000 (actes du colloque à paraître). Il est entendu que je suis seul responsable des idées que je défends ici. Je remercie toutes les personnes qui ont contribué de près ou de loin à cette réflexion, de même que les évaluateurs pour leurs commentaires et leurs suggestions. 
Le corpus étudié est un ensemble d'œuvres et d'événements qui connaissent une sorte de consécration au cours des années 1990. De façon générale, l'activité théorique, critique ou discursive qui accompagne tout le développement du système de l'art depuis la fin du $\mathrm{XIX}^{\mathrm{e}}$ siècle, reconnaît cette tendance forte de l' «intervention », associée à certains mouvements d'avant-garde, qui consiste non seulement à contester les règles et sortir l'art des lieux consacrés, mais aussi à lui faire réintégrer l'espace social, qui sera alors défini selon un axe plus ou moins idéologisé. La forme extrême de cette intégration idéologisée des avant-gardes dans l'espace social est l'art de propagande, par exemple pendant la période révolutionnaire en URSS jusqu'au début des années 1930, soit jusqu'à la dissolution des groupes et des revues autonomes, qui laissent place au dogme du réalisme socialiste. Un moment de cristallisation particulièrement important se produit avec l'Internationale situationniste, puis avec Fluxus, où la pratique de l'intervention s'articule ouvertement autour d'une critique du spectaculaire. La critique de la réification et de l'aliénation, de la nécessité d'une prise en charge et d'une appropriation collective de l'espace social, parcourt aussi d'autres champs de la connaissance (urbanisme, architecture, écologie, mouvements sociaux) qui vont se conjuguer dans les interventions des années 1970-1990.

Cependant, ainsi que le faisait remarquer encore récemment Paul Ardenne (2001) qui donnait au mot «intervention » un sens très large, au point d'amalgamer des pratiques quasi incommensurables comme les démonstrations futuristes des années 1910-1920 et les happenings de Kaprow -, on assiste à une indéniable banalisation de l'intervention dans les années 1970. La charge critique et l'impact des pratiques interventionnistes se diluent, au profit d'une « offre de spectacle » et d'une « demande spectaculaire de surprise », générées dans le cadre d'un système de l'art qui a progressivement homologué et ingéré la plupart des mouvements d'avant-garde et qui s'aligne sur les politiques culturelles de l'État. Le même constat revient régulièrement partout lorsqu'on remet en question les fonctions subversives ou émancipatrices de l'art dans la société contemporaine (Jeudy, 1999; Michaud, 1997). Ardenne indique, par contre, l'existence de formes d'intervention capables de maintenir vivante la volonté d' «interférence », qui parviennent à entraver les processus de dilution ou de dégradation spectaculaire, et qui contribuent à ouvrir, approfondir, intensifier l'espace urbain en tant que lieu de l'expérience sociale.

Une analyse historique sur la longue durée pourrait déterminer, par delà la tendance à la bureaucratisation et à la normalisation institutionnelle (Sioui Durand, 1997), par delà la banalisation ou la dégradation signalée par Paul Ardenne, une tendance inverse qui va vers la recherche d'une alternative, l'invention de «nouveaux territoires » et de «nouvelles pratiques ». Cette nécessité systémique du déploiement de l'art dans la société contemporaine vaut évidemment pour tous les secteurs de la production artistique, et la sociologie propose aujourd'hui au moins deux versions de cette histoire: une version déprimée ou dépressive (Heinich) et une version euphorique ou euphorisante (Luhmann). La solution euphorique, pour peu qu'on prenne garde à ses effets secondaires, est plus fructueuse parce qu'elle prend au sérieux les prétentions à l'autonomie de l'art et ses transformations actuelles. Elle propose également une réflexion sur le sens des œuvres qui n'est pas coupée des questions centrales de la sociologie. Il est certainement plus profitable d'abandonner 
les représentations idéologisées de l'histoire de l'art et de revenir aux propositions artistiques elles-mêmes.

Quelques repères suffiront à identifier plus précisément le genre, à défaut de procéder à une évaluation critique des œuvres singulières. Ce repérage n'est cependant pas simple, et fait déjà intervenir des considérations qui relèvent d'une théorie sociologique plus générale. On pourrait privilégier les événements artistiques collectifs, les expositions thématiques ou les rétrospectives, qui véhiculent explicitement une dimension «montréalaise », et en faire une sorte de série plus ou moins continue, qui ferait état des relations entretenues entre l'art, l'artiste et cet espace urbain particulier qu'est Montréal. On retiendrait par exemple: Montréal tout terrain, Montréal plus ou moins, Corridart, Panique au faubourg, Mémoire vive... Pour s'arrêter un instant sur un moment célèbre de cette série, rappelons que l'exposition Corridart, démantelée par les autorités municipales à la veille de l'inauguration des Jeux olympiques de 1976, proposait un point de vue critique du développement urbain et en particulier de l'histoire de la rue Sherbrooke².

On pourrait tout aussi bien sélectionner des événements qui développent bien une problématique urbaine, mais pas spécifiquement montréalaise, par exemple: L'expérience de la ville, Les commensaux, Gestes d'artistes, La demeure... C'est alors la dimension générique de la ville ou de la mentalité urbaine, plutôt que tel ou tel aspect singulier du patrimoine matériel ou immatériel montréalais, qui est directement interrogé. Gestes d'artistes par exemple, devait initialement se tenir à New York, mais les attentats du 11 septembre 2001 et la « lutte au terrorisme » qui s'ensuivit forcèrent le redéploiement des interventions dans les rues du centre-ville de Montréal. Certains projets sont alors abandonnés, parce qu'ils perdent leur raison d'être, d'autres se transforment, s'adaptent, se transportent analogiquement, en quelque sorte, sans trop de difficultés. Comme l'a dit à cette occasion l'artiste Devora Neumark, dont la performance consistait à entrer en interaction avec les passants de la Place de la Paix en effeuillant des marguerites:

Bien que la spécificité semble différente, la résonance reste la même, en effet. Les gens fréquentant le Christopher Park et ceux qui fréquentent le parc de la Place de la Paix, les gens en général d'ailleurs (et peut-être plus particulièrement les individus qui ont vécu pendant si longtemps en marge, à la limite de l'acceptation de soi et de celle des autres), ont en commun une douleur qui est le résultat du rejet et de l'exclusion. Je reviens donc à cette idée de repenser la vulnérabilité, non pas comme une condition de victime, mais comme une force qui consiste à être présente et engagée, sans construire de barrière pour se protéger de ce qui existe et arrive à l'extérieur. (Traduction, citée dans Fraser et Lafortune, 2001, p. $52^{3}$ )

2. Pour un aperçu général des événements et expositions collectives des années 1970-1980, voir Couture, 2003.

3. «[...] while the specificity is different, the resonance is the same. The people who frequent Christopher Park and the people who frequent the park in Place de la Paix, for that matter everyone (and perhaps especially individuals who have lived for such a long time on the margins, on the edges of self-acceptance and external acceptance), share a similar pain in rejection and exclusion. And so I come back to the question of rethinking vulnerability, not as victimhood but as strength to be present and engaged without building and reinforcing walls of protection from what is inside and out there.» (Citation originale, Fraser et Lafortune, 2001, p. 84) 
Enfin, les œuvres singulières qui sont réalisées dans le cadre de l'un ou l'autre de ces événements, et qui relèvent de la performance, de l'installation, de l'action sociale ou communautaire, de l'intervention, de la manœuvre, les œuvres processuelles ou relationnelles, etc., peuvent déconstruire le spectacle de la ville sans dépendre exclusivement d'un milieu particulier, qu'il soit montréalais ou urbain: ainsi, les interventions de Jean-François Prost ne sont pas moins pertinentes pour notre propos lorsqu'elles se déroulent aux confins de l'espace urbain (terrain vague, friche ou interstice), en milieu rural, voire dans ce qui se présente comme la pleine nature. En février 2000, Prost avait ainsi installé un abri temporaire sur la glace de la rivière Saguenay, en face du village de Sainte-Rose-du-Nord. Intitulé Convivialités électives, le projet poursuivait une démarche entreprise en milieu urbain (Chambre avec vues, en 1998) et où l'artifice techno-architectural cherche avant tout à recréer un espace intime propice au surgissement d'une expérience relationnelle riche et imprévisible. Les terrains vacants ou les espaces résiduels du milieu urbain rejoignent de cette manière les espaces ambivalents de l' «arrière-pays » et ceux réservés aujourd'hui à la «villégiature » pour devenir des occasions d'intervention. Prost s'en explique ainsi :

Je demeure réticent à l'idée d'identifier explicitement mes abris comme des propositions artistiques ouvertes au public. [...] Je ne souhaite pas le rituel de ce type de visite vécue comme une circulation, je veux plutôt privilégier la rencontre. La découverte doit se faire de façon spontanée, inhabituelle et non officielle. La vocation et la fonction de l'abri sont volontairement floues, ambiguës et indéterminées pour intriguer le passant et attirer son attention. Mais tout cela n'exclut pas la possibilité que, sur un site peu fréquenté (au bord d'un strip ou d'un aéroport), je décide de solliciter personnellement certains passants ou automobilistes, ou d'inviter (en collaboration, en complémentarité) un photographe, un vidéaste et un anthropologue à venir passer quelques jours dans l'abri. Ainsi, ce lieu de rencontres est aussi un lieu de séjour et un atelier nomade de production artistique s'inspirant de l'observation du quotidien. (Dufrasne et Prost, 2000, p. 34)

Les artistes qui pratiquent l'intervention urbaine doivent faire face à la difficulté de penser le nouvel espace public et de le concrétiser. Les praticiens de ce genre, qui n'est donc pas si nouveau, puisent librement à diverses traditions artistiques et à diverses technologies; dans mon hypothèse, les artistes sont donc loin d'être des victimes ou des jouets de la nouvelle synergie entre l'État, le marché et la culture: ils en sont les protagonistes et les critiques. Ce n'est pas que l'art se trouve enrégimenté, comme dans la France de la II République (Clark, 1992); il s'agit plutôt d'un art qui se conçoit lui-même comme intervention et stratégie, c'est-à-dire comme un geste problématique, à la fois dans le champ artistique et dans l'espace social, qui aura pour finalité la révélation du fonctionnement social de l'art et la mise en scène du processus de création, d'une part, et la reconversion de l'univers culturel dans les termes d'une pratique émancipée (Huelsenbeck, 1974; Janover, 1989). Cette situation déborde la seule question des avant-gardes, puisque les interventions contemporaines ne s'inscrivent pas dans une logique d'affrontement ou d'escalade révolutionnaire, ni du point de vue formel, ni du point de vue social ou culturel.

Les œuvres dont il est question se distinguent en ce qu'elles entendent déconstruire, déjouer et rejouer le spectacle des villes pour renouer avec le sens du lieu, de l'ici et du 
maintenant, avec le geste en situation et l'interaction, avec les qualités intrinsèques de la pratique, avec la responsabilité de l'artiste, avec le citoyen, etc. De plus, et c'est certainement un des enjeux les plus essentiels comme on le verra en conclusion, ces interventions en milieu urbain remettent en question les catégories usuelles qui permettaient de penser l'espace public.

Une autre façon de caractériser ce corpus particulièrement foisonnant est de s'en remettre à la structure adoptée par Patrice Loubier et Anne-Marie Ninacs pour l'ouvrage Les commensaux, qui couronnait la programmation de l'année 2000-2001 du centre des arts actuels Skol. Les interventions artistiques n'y sont pas identifiées formellement ni classées, mais rapportées, dans les différentes contributions qui composent le volume, à quatre axes d'interrogation - «Infiltration. Circulation. Rencontre. Résistance» -, que les deux auteurs définissent comme suit: «l'investissement de l'espace public, la mobilité de ses frontières et sa fonction communautaire; l'utilisation de l'objet, sa circulation et les protocoles d'échange qu'il génère; l'investigation des relations interpersonnelles, de l'éthique individuelle et de leurs propriétés «matérielles; et, enfin, ce désir [...] de voir s'intensifier le rôle et la fonction critique de l'artiste dans la société» (Loubier et Ninacs, 2001, p. 15). On a là, ni plus ni moins, un programme qui pourrait s'appliquer a posteriori à l'ensemble des interventions artistiques en milieu urbain depuis la fin des années 1970, à ceci près que la tonalité majeure des interventions des années 1990, qui, sur ce point, contraste avec les précédentes, est du côté des micro-utopies et non du côté des mouvements d'émancipation d'inspiration marxiste ou tiers-mondiste. Elles partagent la volonté d'appréhender un aspect ou l'autre de l'espace urbain montréalais, mais elles ne se limitent jamais à une simple reprise de l'imaginaire social: elles n'ont rien de spécialement pittoresque. Bien souvent, et à divers degrés, ces interventions sont ouvertement antispectaculaires, ou alors elles prétendent échapper au spectaculaire par la ruse.

\section{LA REVITALISATION URBAINE}

Le champ de l'intervention artistique en milieu urbain, avec ses dynamiques propres et ses contradictions internes, émerge de façon claire à la fin des années 1970 avec la restructuration du marché immobilier due au déclin des centres et des anciennes zones industrielles, les interventions des États et des municipalités en matière d'infrastructure culturelle, les programmes de réhabilitation, de revitalisation et de revalorisation urbaine. La « reconquête culturelle », ainsi que l'appelait un document du ministère français de l'Aménagement du territoire et des Reconversions (1991), est conçue comme un ingrédient indispensable du développement économique et de la mise en scène internationale de la ville. Avant de définir plus précisément le champ des interventions artistiques en milieu urbain, il importe de saisir cette réalité du développement culturel.

À l'automne 2003, deux projets de revitalisation urbaine relançaient la discussion sur le rôle de l'art et des artistes dans la vie culturelle des Montréalais. Il s'agissait de la construction d'une maison de la culture sur la place Valois, dans le quartier HochelagaMaisonneuve, et du projet Quartier des spectacles, dans le centre-ville. Dans le cas de la place Valois, pour une deuxième année consécutive, des artistes s'étaient regroupés pour 
organiser un événement d'art public intitulé Urbaine urbanité II : une maison de la culture virtuelle et nomade $e^{4}$ Les artistes avaient disposé des conteneurs sur un terrain vague laissé par la démolition d'un ancien garage. Les conteneurs faisaient office de salle d'exposition et de dispositif artistique. Une maison mobile accueillait les visiteurs et servait de bureau d'information. Les instigateurs de l'événement entendaient à la fois protester contre un certain type de développement urbain, exprimer la vitalité de la recherche et de l'expérimentation en art, favoriser des activités de collaboration entre les artistes et la communauté, et repenser le rôle traditionnel de la maison de la culture. Dans le communiqué diffusé à cette occasion, on lisait notamment:

C'est tout un quartier qui connait de profondes transformations, tant démographiques et économiques, que sociales et culturelles. À l'heure où la Ville de Montréal s'engage dans la définition d'une nouvelle politique culturelle, et où la configuration, voire le statut même du quartier, est l'objet de discussions entre les citoyens, les élus, les gestionnaires municipaux et le gouvernement provincial, il est nécessaire de s'interroger sur le rôle des institutions et des équipements culturels, et en particulier sur le réseau des maisons de la culture et la place que revendiquent les artistes dans l'espace public. Des définitions concurrentes et parfois contradictoires de la culture sont mobilisées par les uns et les autres, des définitions qui oscillent entre les exigences de la démocratisation, celles de la participation, celles des artistes qui cherchent de nouvelles voies de création, de diffusion et d'intervention, celles des décideurs qui entendent faire de la ville une métropole culturelle, stimuler la production, développer de nouveaux publics, mettre en valeur le patrimoine ou favoriser les échanges interculturels. De larges franges de la population sont toujours exclues, tenues à l'écart ou marginalisées, et ne bénéficient pas du tout ou très indirectement du dynamisme culturel du quartier.

Quelques mois plus tard, on apprenait qu'un autre projet d'immobilisation, toujours dans le quartier mais non Place Valois, était examiné par le ministère des Affaires municipales. Ce projet verrait la création d'une maison de la culture dans l'ancienne caserne de pompiers, un bâtiment converti en salle de spectacle et laissé vacant après la faillite de l'organisme Faites de la musique!

La question de la prise en charge du développement culturel du quartier par les citoyens et les groupes communautaires ou artistiques n'est pas nouvelle, elle était même présente à l’origine du réseau des maisons de la culture de la Ville de Montréal il y a plus de vingt ans. La question, comme le dit le communiqué, mobilise des conceptions de la culture et surtout des manières de faire très différentes; la participation et l'esprit communautaire, d'une part, la production et le développement, de l'autre... et entre ces deux

4. L'événement était organisé par l'artiste Gilles Bissonnet et la galerie FMR, et s'est déroulé du 12 au 20 septembre 2003. Les artistes participants étaient: Gilles Bissonnet, Johanne Chagnon, Pierre Crépô, Farine orpheline cherche ailleurs meilleur, Paul Grégoire, Aude Moreau et Armand Vaillancourt. Une table ronde animée par Gilles Bissonnet et moi-même réunissait Ariane Émond, directrice générale de l'organisme indépendant Culture Montréal, Michel Roy, représentant des groupes communautaires du quartier, Jean-Claude Laporte, du Comité bails, et Philippe Côté, artiste. Dans une vidéo tournée sur place et projetée en différé, le sociologue et critique d'art Guy Sioui Durand proposait ses réflexions sur le rôle de l'artiste dans l'espace public. 
principes d'action, les efforts des uns et des autres, citoyens, artistes et professionnels de la culture, qui inventent ou qui cherchent à se réapproprier la culture réelle.

Un autre type de tension entre conceptions différentes de l'action culturelle se manifestait dans le cas du Quartier des spectacles. Ici, on était en présence d'un projet à vocation à la fois culturelle, commerciale et touristique. Selon une étude de préfaisabilité publiée en 2001, le projet initié par l'Association québécoise de l'industrie du disque, du spectacle et de la vidéo (ADISQ) et le promoteur-producteur Spectra-Scène ferait l'objet d'une concertation entre propriétaires de salles, les milieux montréalais du spectacle vivant et les « commerces connexes » (restaurants, petits hôtels, cafés, galeries) implantés dans le quartier ou désireux de le faire. Il s'agirait de s'appuyer sur les salles bien établies du secteur (les Foufounes électriques, le Spectrum, le Club Soda, le Métropolis, le Cinéma Impérial) et les organismes importants tels que la Place des arts, le futur complexe gouvernemental de l'îlot Balmoral et l'Orchestre symphonique de Montréal, pour offrir « un vaste choix de spectacles sur scène, accessibles à pied », dans un quartier « agréable, animé et sûr» (cité dans RCAAQ, 2003). À ce discours, qui s'articule autour de la promotion de l'industrie du spectacle et du tourisme et qui favorise le rayonnement international de la ville «dans des buts de culture et de divertissement», s'opposait celui du Regroupement des centres d'artistes autogérés du Québec. Dans un document déposé à l'occasion des audiences publiques de la Commission d'urbanisme de la Ville de Montréal, le Regroupement défendait la vitalité et la pertinence d'un tout autre univers, celui de l' «art actuel» ou de l'«art contemporain». Douze centres d'artistes sont établis dans le quartier, des organismes en arts médiatiques, des revues d'art, d'importantes galeries ainsi que le Musée d'art contemporain. En plus d'assurer la production et la diffusion des secteurs de pointe en arts visuels et médiatiques, du cinéma et du théâtre d'avant-garde, les artistes implantés dans le quartier ont directement participé à sa requalification depuis dix ans et créé une synergie avec les universités voisines (Université du Québec à Montréal, Concordia, McGill). Le Regroupement s'inquiétait qu' « un projet à vocation culturelle nie, oublie ou fasse disparaître cette fourmilière de création » et appelait une certaine complémentarité entre art actuel et industrie du spectacle (RCAAQ, 2003). Conscient des dynamiques du développement urbain, et en particulier du processus de gentrification qui menace de faire migrer à nouveau les centres d'artistes, le Regroupement concluait ainsi :

La vocation du spectacle qui s'accompagne [...] d'une agitation propice à rameuter les foules de spectateurs nécessaires à sa survie ne doit pas primer sur tout autre aspect de la vie artistique montréalaise. C'est pourquoi nous tenons à rappeler aux promoteurs du Quartier des spectacles ces complémentarités dont ils devront tenir compte s'ils ne veulent pas nous laisser un centre-ville vulgaire, uniquement parcouru par des foules à la recherche du dernier Disneyland.

La sociologie de l'art a tenté une explication de ce phénomène récurrent en cherchant du côté des transformations structurelles et démographiques de la ville, qui se traduisent notamment par la gentrification. Le processus de gentrification est bien connu, et son lien avec la dynamique du monde de l'art a été établi il y a longtemps. Ainsi que l'expliquait notamment Sharon Zukin (1982), la mode des lofts, apparue au début des années 
1970 à Amsterdam, Londres ou New York, se conjugue au capitalisme postindustriel et au changement social plus général qui affecte les villes forcées de réorienter leur développement. Ce type de développement où des artistes combinent atelier et résidence dans des locaux industriels abandonnés, avant de céder la place à d'autres réseaux professionnels ou d'autres entreprises capables d'accompagner la spirale inflationniste, pourrait avoir commencé dès la fin des années 1950 dans SoHo, selon Charles R. Simpson (1981). Quelles que soient les caractéristiques de chaque quartier ou de chaque ville, le processus est à peu de chose près le même partout. Et c'est précisément ce mouvement qui a provoqué la prise de position du Regroupement des centres d'artistes autogérés du Québec, non pas tellement pour défendre le sort réservé à des ateliers ou à des résidences, mais pour réaffirmer de manière positive la présence active de quelques-uns de leurs membres au cœur de ce quartier qu'on entendait transformer, de façon typiquement spectaculaire, sans prendre en compte la contribution, la mission et les objectifs spécifiques des centres d'artistes. Reste à savoir si le nouveau partenariat créé en juin 2003 et incluant en outre des représentants des résidants, des milieux artistiques et d'une institution comme l'Université du Québec à Montréal pourra donner au projet de Quartier des spectacles une autre orientation.

Il serait dommage que la critique assurément pertinente du capitalisme flexible postindustriel et de ses effets sur la production de l'espace urbain masque les conflits d'interprétation qui entourent la définition de l'espace public et la signification des interventions artistiques en milieu urbain. Zukin faisait valoir que le développement de la production artistique et de la consommation culturelle est lié à des changements dans le marché immobilier et aux projets de conservation ou restauration du patrimoine dans les centres; une synergie fragile et temporaire, une vitrine de prestige pour attirer les investisseurs et les grandes organisations (soit de manière inattendue comme dans SoHo, soit de manière très planifiée comme dans les Docklands de Londres ou Battery Park City à New York). Mais sur ce point, elle restait bien en deçà de ce qu'on peut attendre d'une sociologie de la production artistique (voir Richard A. Peterson dans Crane, 1994, p. 163-189), et surtout ignorait la perspective compréhensive que j'avancerai plus loin.

\section{Les POlitiques CULtURELles}

Une autre explication de l'émergence de ce champ et de ce genre particulier qu'est l'intervention artistique en milieu urbain peut être trouvée du côté des politiques culturelles. En dépit de la grande diversité des situations, une série de tendances communes encouragent les gouvernements municipaux à augmenter leur part des dépenses et leur présence dans l'offre de services culturels; que ce soit en vertu de politiques nationales de décentralisation des pouvoirs, des nouvelles demandes culturelles des associations locales et des mouvements sociaux, ou plus largement pour se conformer à une certaine idée de la restructuration de l'économie.

Dans plusieurs pays occidentaux, l'action culturelle de l'État trouve en effet sur les plans municipal et régional un espace privilégié pour des projets dits structurants, notamment dans la réhabilitation ou la revitalisation urbaine, le développement et l'aménagement du territoire (Thuriot, 1999). Cela est particulièrement frappant au Québec et à 
Montréal en particulier, où la décentralisation et l'attribution de certaines compétences aux collectivités locales, de nouvelles formes de partenariat ou de coopération, ont des effets directs sur l'évolution des espaces ruraux et urbains. Dans l'œil des promoteurs, les fonctions sociales de l'art et de la culture sont définies selon des axes convenus: la culture est perçue comme un facteur d'identité et de solidarité, un élément indispensable à la requalification des quartiers ou à la lutte contre l'exclusion sociale. La culture et la production artistique contemporaine peuvent aussi apparaître comme des adjuvants du rayonnement de la ville ou de la région sur le plan international ${ }^{5}$.

Cependant, comme de nombreux observateurs l'ont établi, tant en Europe qu'en Amérique (D’Angelo, Freidberg et Urfalino, 1989; Blau, 1989 ; Crane, 1987; Latouche, 2003), le rôle des villes en matière d'art n'est pas monolithique. Les décideurs et les appareils administratifs sont traversés par des logiques de développement, des systèmes d'alliance et des stratégies, des attentes pas toujours faciles à concilier, sans parler des pressions de l'opinion publique et de la société civile. À Montréal comme ailleurs, mais peut-être avec une acuité accrue à cause d'une politique de restructuration municipale très contestée (Nielsen, Hsu et Jacob, 2002), les logiques de la « démocratisation» et de la «démocratie culturelle » n'avancent pas toujours de façon complémentaire. Ainsi, le partage des compétences ou des responsabilités entre les arrondissements et l'administration centrale peut aller dans le sens contraire des décisions prises il y a moins de quinze ans, la logique de la professionnalisation et de l'autonomisation du secteur culturel semblant reculer ou marquer le pas face à la logique de la participation et de la décentralisation (Bellavance, Santerre et Boivin, 2000).

Ainsi que le rappelait Franco Bianchini, dans son étude de quelques politiques culturelles européennes depuis les trente dernières années, des dilemmes cruciaux apparaissent ou s'aggravent: les disparités entre la ville-centre et sa périphérie (gentrification, ségrégation, problèmes d'intégration socioculturelle et économique); les conflits entre les stratégies orientées vers la consommation, les services, les attractions, qui créent souvent des emplois peu qualifiés, d'une part, et les stratégies orientées vers le soutien à la production, qui demandent des investissements réfléchis, d'autre part; entre les investissements dans les infrastructures et les équipements lourds et les investissements plus ponctuels ou éphémères dans les activités culturelles locales (Bianchini et Parkinson, 1993). À Montréal, le démantèlement de La joute de Jean-Paul Riopelle, une œuvre d'art public réalisée à l'occasion des Jeux olympiques de 1976, et sa relocalisation dans le nouveau quartier des affaires et du multimédia en 2003 illustrent de façon criante les dilemmes de l'action de l'État provincial et de la municipalité en ce domaine, partagée entre des conceptions différentes de l'art, du développement, de la démocratie (Doucet, 2003; Beaudet, 2003). En somme, les

5. Ces idées étaient exprimées très clairement dans le rapport du groupe-conseil présidé par Raymond Bachand (Ville de Montréal, 2003a). Le groupe, un organisme indépendant composé de personnes issues des milieux culturels, avait comme mandat de définir les grandes lignes d'une nouvelle politique culturelle pour la Ville de Montréal. Le rapport se penche sur le rôle de la Ville en matière de culture, mais également sur la contribution de la culture à la vie de la cité sur les plans régional, national et international. Il propose notamment quelques pistes d'action, dont l'amélioration du réseau des bibliothèques et le soutien à la création. Le dépôt du rapport est assorti d'un processus de consultation publique. 
actions publiques qui visent à requalifier le milieu urbain ravivent des controverses importantes liées à l'espace public et à l'expérience de la ville. Il est vrai qu'elles courent aussi le risque parfaitement banal, comme les œuvres et les interventions artistiques ellesmêmes, de disparaître dans le bruit ambiant...

\section{L'INSERTION DES GUVRES DANS LEUR MILIEU}

Mais un examen attentif des tensions qui définissent l'action culturelle officielle ne dit pas encore ce qu'est le sens des interventions artistiques en milieu urbain. Comme l'a vu Joost Smiers (1998), il faut bien sûr prendre en compte à la fois les obstacles et les incitations à la création; le rôle des animateurs et de la critique indépendante; les blocages de la diffusion; la présence des industries culturelles et la concentration des médias. Sur ce point, il faut aussi prendre en compte l'omniprésence de formes plutôt décevantes, voire finalement assez pauvres de la participation dans le domaine de la production et de la création culturelles: reality show, focus group, scénario soi-disant interactif, vox pop, etc. Mais ces obstacles - la démocratie postmoderne et l'instrumentalisation de l'espace public - ne sont peut-être pas si graves, puisque la citoyenneté implique bien davantage que la seule formation de l'opinion publique et la participation aux mécanismes de la démocratie de masse.

Pour anticiper un peu sur ce qui sera dit plus loin, c'est peut-être là que se situent l'originalité et la fragilité des interventions artistiques: à la différence du produit sur mesure ou conforme aux attentes ordinaires, à la différence des programmes officiels de revitalisation, l'intervention veut ménager un espace de "dessaisissement », d' " extranéation », de distanciation... en dépit et par delà les contraintes librement consenties de la culture, de la société, du politique. Jean-François Augoyard le disait en ces termes: «l'action artistique urbaine n'est jamais indifférente aux composantes humaines et physiques de la ville» (Augoyard, 2000, p. 19). La définition provisoire qu'il en donne peut nous être utile ici. L'action artistique urbaine désigne selon lui «toute action-création émanant d'une expression artistique qui compose avec l'espace construit d'une ville et appelle un rapport spécifique avec le public urbain » (ibidem). C'est donc dire que les problèmes de la forme et du contenu de l'intervention sont d'emblée hétéronomes et ouverts sur des dimensions extra-artistiques. Augoyard souligne comment, dans ce contexte, l'intervention participe à une redéfinition de l'espace public, notamment en engageant « une modification de la perception et de la représentation de l'espace-temps quotidien» :

l'espace public n'est pas seulement le support de fonctionnalités essentielles, ni seulement l'entre-privé régi par le principe d'accessibilité, ni seulement l'aire des observables réciproques fondant les interactions sociales concrètes. Il est aussi objet de jeu, de dramatisation, de plaisir et d'expression des subjectivités [...] L'action artistique urbaine n'a pas à produire des effets en fonction d'objectifs fonctionnels et extrinsèques. Son sens est de manifester la création comme acte signifiant, comme ouverture sur le possible au sein d'un univers de fonctions et d'objets donnés. (p. 18, 20)

L'invocation du jeu et de la subjectivité n'est certes pas un critère suffisant, mais indique l'orientation générale d'un acte spécifique dans l'espace public. Un autre observateur 
attentif de ce phénomène, Fabrice Raffin (2000), a insisté quant à lui pour signifier que la question de l'inscription urbaine des actions culturelles et des pratiques artistiques (notamment l'occupation de friches industrielles, usines, bâtiments, garages, appartements ou locaux abandonnés, squat) doit se poser au-delà des invocations du discours public et de ses stratégies, pour rejoindre les différents registres d'un milieu social spécifique. On analyse alors l'action en termes de processus incluant une grande variété d'acteurs (les intervenants professionnels, les publics, les riverains, etc.); on prend en compte les contraintes et les transformations d'un espace donné, ainsi que les formes d'apprentissage et les modes de vie qui y sont liés. La redéfinition de l'espace public en effet passe par chacun des moments de la conception et de la réalisation des œuvres qui, comme on l'a déjà signalé, exigent une forme ou une autre de participation, de coopération, d'interaction.

Quoi qu'il en soit, au nombre des éléments systémiques qui déterminent les pratiques artistiques interventionnistes, il faut certainement accorder une attention particulière à des politiques plus conjoncturelles. Depuis quelques années, la Ville de Montréal cherche par exemple à affirmer son rôle sur le plan de la conservation et de la mise en valeur de sa collection d'art public, à l'instar des autres villes nord-américaines. Or, les discussions qui ont cours sur les plans municipal et provincial ont trait aussi à l'opportunité de soutenir plus directement des formes éphémères d'art public, en rupture par rapport aux habituels concours, en favorisant des interventions sur le mobilier urbain, des expositions temporaires, des événements, des expériences d'art communautaire et d'animation culturelle. C'était par exemple le cas, entre mai et octobre 2000, avec l'événement D'un millénaire à l'autre qui proposait des expositions multidisciplinaires dans onze maisons de la culture, et une série d'installations et d'interventions en plein air réparties dans des parcs, des places et sur le mobilier urbain ${ }^{6}$.

Jusqu'à présent, les artistes qui pratiquent l'intervention ont surtout développé la faculté de contourner ou de se concilier les nombreux obstacles d'ordre législatif et réglementaire qui ponctuent la réalisation de leurs projets. Il apparaît que la réalisation d'un projet artistique est d'emblée posée comme un processus de coopération ou de négociation qui force un dialogue entre le savoir des experts (artistes ou fonctionnaires), celui des publics, celui des usagers et des citoyens, regroupés ou non ${ }^{7}$. Comme l'écrivait Lise Lamarche, en empruntant le terme à René Lourau, les œuvres deviennent des «analyseurs», des révélateurs dont la seule existence ou le seul déploiement dans l'espace public produit une «analyse de la situation» (Lamarche, 1999, p. 286).

\section{LES PROFESSIONNELS DE L'INTERVENTION}

Sur le plan du statut de l'artiste, l'intervention correspond à une figure particulière, aux antipodes du génie saturnien. L'artiste est un professionnel, un chercheur, parfois un

6. Le volet «installations extérieures temporaires » réunissait le collectif d'artistes BGL, Gilles Bissonnet, Trevor Gould, Rose-Marie Goulet, Diane Landry, Marc Larochelle, Devora Neumark, Roberto Pellegrinuzzi et Michel Saulnier.

7. Sur la dimension participative des interventions artistiques, voir mon article: «Les pratiques artistiquescitoyennes: processus ou politique? », Esse, n $^{\circ}$ 48, été 2003, p. 20-25. Je reviens sur cette question plus loin. 
travailleur de l'ombre qui reste derrière le rideau ou simplement l'initiateur d'un processus qui restera anonyme. Bien que le statut de l'artiste professionnel, sanctionné par les lois de 1987 et 1988, puisse à son tour être contesté par l'artiste qui entend rompre avec le système de l'art, les institutions ou les pratiques dominantes, il reste que cette caractéristique organisationnelle du monde de l'art contemporain doit être prise en considération pour saisir le sens des interventions urbaines.

La nouvelle figure de l'artiste qui se conçoit lui-même comme un acteur social (artiste engagé, militant ou animateur, anthropologue, intervenant, etc.) est bien entendu préparée par des décennies de pratiques d'avant-garde (pour la période des années 1960-1970, voir en particulier Couture, 1999). On remarquera aussi, ainsi que le faisait déjà la sociologie des professions appliquée aux secteurs artistiques en général (Freidson, 1987; Menger, 1989, 1999), que les professionnels de ce monde particulier qu'est celui de l'intervention en milieu urbain ont en commun un haut degré de polyactivité et un attrait certain pour ce que Menger a appelé le «risque», l'«imprévisibilité» ou l'«incertitude de la vie d'artiste».

Les indices dont nous disposons sur les traits socioprofessionnels de la population d'artistes qui produisent des œuvres destinées à l'espace public urbain laissent penser qu'il s'agit de la frange la plus scolarisée, la plus polyvalente et la plus active (voir par exemple Bellavance, Bernier et Laplante, 2001). Les professionnels interrogés dans le cadre de la présente étude sont à la fois artiste et chercheur, commissaire et historien, agent culturel et commissaire, ou critique et commissaire, etc. Ces personnes occupent des fonctions tantôt officielles tantôt parallèles ou excentriques et cumulent les charges. Mais le plus important à noter est qu'elles sont toutes animées par la logique de l'engagement ou de la vocation, à quelque niveau qu'elles agissent, et font preuve nécessairement d'un maximum d'inventivité dans l'exercice quotidien de leur tâche. Ce polymorphisme et cette créativité s'étendent maintenant à la capacité d'initier des projets et de les mener à terme, même si l'artiste n'est plus qu'un élément parmi d'autres dans le processus de réalisation, même si l'«auteur» peut s'éclipser totalement au bénéfice des pratiques et des discours sociaux qu'il ne maîtrise pas et qui font partie intégrante de l'intervention.

Autre élément conjoncturel important: la création de programmes de bourse et de subvention destinés à des projets et à des événements multidisciplinaires. La création du Bureau Inter-arts au Conseil des Arts du Canada est aussi à prendre en considération, programme qui favorise notamment la tenue d'événements spéciaux à caractère novateur comportant un volet théorique. Mais ces changements du côté des programmes officiels, et la volonté affichée par plusieurs centres d'artistes et par plusieurs intervenants de dépasser les frontières disciplinaires, n'ont pas encore donné beaucoup d'exemples concrets de coopération entre les arts visuels et médiatiques, le design, l'architecture, l'urbanisme et les communications.

De façon plus générale, le mode de financement des organismes artistiques et en particulier des centres d'artistes autogérés depuis la fin des années 1970 a pu favoriser la multiplication des interventions en milieu urbain; en effet, une pression de plus en plus grande a été exercée pour que les organismes se tournent vers des projets ponctuels, en partenariat, pour le développement de nouveaux publics. Les mêmes politiques ont également favorisé la multiplication d'événements en dehors des grands centres urbains, accentuant 
encore le phénomène de la circulation et de la fluidification des frontières entre les centres urbains proprement dits et les régions, la ville et la campagne, la ville et la nature (voir par exemple Danyèle Alain et al., 1999, 2000, 2002 ; Fortin, 2002).

Il se produit par ailleurs un mouvement typique de convergence des actions de différents ministères et organismes culturels ou artistiques qui se préoccupent de la ville, parallèlement au climat entretenu par les grands médias en faveur de la poignée de festivals qui se déroulent dans l'espace urbain. Même s'il ne crée pas un authentique intérêt pour les questions urbaines ou patrimoniales, comme le voudraient les groupes de pression et les comités de citoyens, ce mouvement de convergence organisationnel est un autre élément systémique qui conditionne les interventions artistiques, ne serait-ce qu'en leur donnant une bonne raison d' « interférer » dans le spectacle de la ville, voire d' « entraver » le spectacle.

\section{L'ART EN MILIEU URBAIN ET LES NOUVEAUX ESPACES PUBLICS}

La notion d'espace public fait référence à une organisation formelle et politico-institutionnelle de la société moderne, aujourd'hui en pleine mutation (Bauböck, 1992; Bourque, Duchastel et Pineault, 1999; Habermas, 1997). Cette mutation n'a bien entendu pas que des effets sur la société civile et les structures politiques, elle en a aussi sur les droits sociaux et culturels associés à la nouvelle citoyenneté, et finalement sur tous les aspects de la représentation, de l'expression, de la dialectique des identités dans les sociétés pluralistes (Araeen, 2000; Bharucha, 1999; Elbaz et Helly, 2000 ; King, 1997 ; Kymlicka, 1995; Zizek, 1997). On est en droit de parler de nouveaux espaces publics, puisqu'ils excèdent aujourd'hui, tant sur le plan architectural que sur le plan institutionnel, les formes traditionnelles. L'espace public en effet se traduit ou se manifeste sur le plan architectural bien au delà de la place publique - par ailleurs très balisée ou contrôlée - pour se couler dans les canaux de communication et de diffusion, dans la moindre des ramifications du tissu urbain construit, dans les zones interstitielles, voire sauvages, comme dans les relations intersubjectives informelles qui appartenaient il n'y a pas si longtemps encore au monde de la vie privée ${ }^{8}$.

Joost Smiers (1998) observait que les espoirs mis dans l'animation populaire et la revitalisation-revalorisation des quartiers dans les années 1960, 1970 et 1980 ont été presque partout vaincus par l'essor des médias de masse, l'idéologie de la réussite, le spectaculaire, les promoteurs et les investisseurs. Dans ce contexte, l'existence d'une variété de lieux d'expression et de réflexion dans l'espace public est menacée par l'emprise du commerce et des principes d'organisation d'un certain urbanisme policier. Il nous faudrait comprendre comment de façon générale la création artistique engage une modification des représentations et des usages liés à l'espace public (voir aussi Augoyard, 2000).

8. En 2003, la galerie Monopoli invitait seize créateurs issus des milieux de l'art public, du design urbain, de l'architecture et du paysage à répondre à la question : «Y a-t-il encore, au xxi ${ }^{\mathrm{e}}$ siècle, des places réellement publiques?» Chaque proposition devait adopter le format d'une «boîte» de 41 x $61 \mathrm{~cm}$, pour être exposée dans la galerie; les «boîtes» ont été exposées à nouveau dans le cadre de la 4 e Biennale de Montréal de l'automne 2004, dont le thème était "Agora : le domaine public ", et qui s'interrogeait notamment sur les transformations actuelles et potentielles de l'espace public. 
Les nouveaux espaces et les nouveaux médias d'intervention que ne cessent d'engendrer les pratiques dites d'art public posent des problèmes méthodologiques et herméneutiques inédits. Dans certains types d'intervention artistique, comme d'ailleurs en architecture et en urbanisme, l'espace lui-même apparaît moins comme une donnée objective, une dimension plus ou moins maîtrisée et exploitée, et de plus en plus comme un artifice humain et technologique, une réalité évolutive, un effet ou une conséquence du territoire imaginé.

Dans l'ensemble du corpus «intervention artistique en milieu urbain», chaque œuvre prend en compte la dimension symbolique et pratique de l'espace. J'y ai fait allusion plus haut en évoquant le travail de Devora Neumark et de Jean-François Prost, ou un événement comme Urbaine urbanité. J'ai proposé ailleurs une analyse de l'intervention Hypothèses d'amarrages ${ }^{9}$, qui montre comment la disposition de tables à pique-nique d'apparence anodine dans des lieux inusités peut laisser place à des relations sociales inédites et susciter un nouveau regard sur la ville. Un seul autre exemple, que je développerai un peu longuement, suffira à illustrer cette prise en compte de l'espace dans les interventions artistiques. À la différence des cas cités plus haut, celui-ci ne comporte pas une dimension relationnelle ou performative très appuyée, mais il n'en est pas moins pertinent. Il s'agit d'une installation de Marcus Macdonald, Proposition pour trois tunnels, réalisée en collaboration avec Sophie Herché et Frank Légale, qui occupa, pendant trois semaines en octobre 1999, un ancien tunnel près du Vieux-Port de Montréal, le tunnel Wellington, aujourd'hui abandonné et en ruine ${ }^{10}$. L'artiste et ses collaborateurs avaient délibérément décidé du lieu, qui n'est pas à proprement parler un lieu ou un espace public. Mais comme tout ouvrage d'ingénierie, il traduisait en quelque sorte les intérêts des commerçants, des industriels et des autorités publiques qui façonnent l'espace public urbain dans son ensemble, et ce très massivement depuis le $\mathrm{XIX}^{\mathrm{e}}$ siècle, comme en témoigne en outre aussi le design des parcs ou des gares. Aujourd'hui, le quartier est écartelé entre différents programmes de développement et l'idée de conservation du patrimoine, entre les besoins des résidants et ceux des investisseurs, entre les vocations parfois contradictoires qu'on souhaite redonner au canal de Lachine. Le tunnel Wellington et le canal qu'il traverse ne sont pas des objets architecturaux uniques qu'on pourrait abstraire de l'ensemble dans lequel ils s'insèrent et prennent sens.

L'ensemble du site choisi par Marcus Macdonald attire l'attention du visiteur, même si ce dernier n'a aucune connaissance historique ou architecturale particulière: la courbe très prononcée et la descente, ou l'approche du tunnel sur la rue Wellington ; certains éléments décoratifs de l'ouvrage (frise, parapet); mais surtout les imposantes grilles cadenassées qui bloquent l'entrée ou les entrées du tunnel (il est en fait constitué de quatre

9. «Dans l'attente de vivre: à propos des Hypothèses d'amarrages de Luc Lévesque et Jean-François Prost ", dans Patrice Loubier et Anne-Marie Ninacs (dir.), Les commensaux: Quand l'art se fait circonstances, Montréal, Centre des arts actuels Skol, 2001, p. 47-50.

10. Je reprends certains éléments d'un texte inédit, «Le théâtre sonore de Marcus Macdonald : une intervention artistique dans le discours de la ville », présenté dans le cadre du colloque «Identité, appropriation culturelle et postcolonialisme» du Comité de recherche en sociologie de l'art, Congrès de l'AISLF, Québec, 3 juillet 2000 . 
voies). La nuit, le site est éclairé par des projecteurs puissants. L'endroit est parsemé de quelques sculptures d'aspect brut, rouillées, de petite dimension; on ne sait trop s'il s'agit d'objets trouvés ou détournés. Des bancs publics, parfaitement incongrus à cet endroit, sont disposés étrangement dans la courbe de la rue, la disposition évoquant parfois l'hémicycle d'un théâtre, mais l'impression d'ordre et de désordre est plutôt inquiétante. La clameur de la ville est partout présente.

Mais ce sont certainement d'abord les sons étranges qui émanent du tunnel qui retiennent l'attention, on peut les entendre bien avant d'apercevoir le tunnel. Ces expériences éparses provoquées par l'ouvrage d'ingénierie en ruine et par les interventions de l'artiste sont subordonnées à un environnement sonore ou à un théâtre sonore. Il s'agit de bruits très connotés, construits pour évoquer des images ou des sentiments, un peu comme la bande sonore d'un film. Macdonald investit trois des quatre voies du tunnel, la quatrième étant occupée par une bouche d'aération. Le premier tunnel produit des vibrations infra-basses et basses, qui agitent l'eau d'un bassin semblant plonger vers le fond du tunnel; dans le deuxième, une voix féminine chante et parle dans une langue inventée, laissant échapper des sons troublants, des mots pas toujours compréhensibles; dans le troisième, un ensemble diffus de sons d'un registre aigu, des sifflements, des grincements. Les trois décors sonores sont générés par ordinateur et de façon aléatoire, à partir de sons échantillonnés ou produits par un synthétiseur, et diffusés au moyen d'enceintes acoustiques dissimulées au fond de chacun des tunnels.

Le tunnel barré est devenu une caisse de résonance dont on ne connaît pas le fond; un antre, un gouffre. Macdonald, tel qu'il s'en explique, a voulu donner un fondement mythologique à l'installation. Pour l'artiste, et certainement pour beaucoup de visiteurs, le tunnel peut représenter à la fois la matrice maternelle, les grottes initiatiques, les passages labyrinthiques d'une ville fortifiée, les catacombes ou, par contiguïté avec le canal, les eaux du Styx qui mène au royaume des morts - bref, le monde où règne la nuit avec ses secrets intimes, ses vérités et ses frayeurs. L'artiste aurait donc choisi d'intervenir dans les registres de l'intimité, mais bien entendu cela ne détermine pas absolument le sens de l'œuvre ou l'expérience que tout un chacun pouvait en faire à l'automne 1999. Chacun des spectateurs est quasi spontanément porté à associer les sons à des sentiments ou à des actions, comme nous invite à le faire tout le dispositif: les trois tunnels agissent en effet comme des tableaux visuels et sonores, ils symbolisent quelque chose qui n'est pas donné directement dans leur seule présence mais qui néanmoins se manifeste dans l'expérience esthétique que nous en faisons, accompagnée de sentiments ou d'émotions que nous connaissons bien ou croyons reconnaître. Des critiques, suivant en cela le propos de l'artiste, ont par exemple associé les tableaux à un triptyque qui représenterait les cycles de la vie, de la vie intra-utérine à une expérience de mort approchée (near death experience), en passant par l'enfance, par l'apprentissage du langage humain et des émotions. La performance de la chanteuse Sophie Herché qui se produira à quelques reprises va relancer encore cette herméneutique dans d'autres directions, notamment en évoquant les immigrés irlandais qui ont vécu et travaillé aux abords du canal de Lachine depuis le xıx ${ }^{\mathrm{e}}$ siècle.

Après la surprise et le désir de reconnaissance, on peut tenter une écoute active de l'installation. Or, le dispositif technique vient contraindre ou limiter la processualité de 
l'écoute. On va constater toutes sortes de coïncidences et de perturbations lorsque les trois tableaux se conjuguent ou tendent à se conjuguer à nos oreilles; mais on n'écoute pas de la musique, on ne peut pas abandonner l'idée qu'il s'agit d'une proposition sonore forçant un retour au contexte pragmatique de l'installation. Comme l'indique son titre, l'installation est effectivement une proposition, une intervention pragmatique dans les discours sociaux qui façonnent l'espace urbain. Il ne s'agit pas d'une opposition simple: le dispositif nous invite à voir et à revoir autrement le quartier, son identité et son histoire, à entendre et à porter attention à l'infinité de sons ou de voix qui le composent.

La quête de lieux alternatifs par les artistes hors norme, innovateurs, ou ceux dont l'insertion professionnelle est bloquée - cette quête ou cette fuite en avant qui les pousse vers des friches industrielles, des terrains vacants, des bâtiments ou des locaux abandonnés, des lieux inusités ou méprisés, et par laquelle ils tentent avec plus ou moins de succès de décloisonner les pratiques et les publics, de s'intégrer à un quartier, un milieu social, de s'immiscer plus profondément dans la culture quotidienne -, fait face aux contraintes de l'institutionnalisation et de la médiatisation. La trajectoire de l'organisme Quartier Éphémère, qui avait invité Macdonald, est à cet égard semblable à celle d'autres centres autogérés. Lieu de création et de diffusion pour la relève créé en 1994 sur le modèle des Usines éphémères en France, il concluait en 1995 une entente avec la Société de développement de Montréal qui lui permettait d'occuper et d'aménager un immeuble abandonné, un ancien entrepôt de métal de rebut. En 1997, l'immeuble était détruit par un incendie, et l'organisme emménageait plus à l'est, dans la rue Peel, avant de se relocaliser, au terme de l'entente de cinq ans, dans l'ancienne fonderie Darling, pour y ouvrir enfin un centre d'art et concevoir de nouveaux projets.

Le regard que je porte sur les pratiques artistiques en milieu urbain ne vise certainement pas à découvrir en elles les nouveaux champions de l'espace public postmoderne ou de l'État cosmopolitique universel, ce qui serait absurde, mais à comprendre les pratiques et les œuvres dans leur tension avec le milieu, et, comme la matérialisation problématique d'une certaine idée de l'expérience de la ville, du mouvement des personnes et de leurs sentiments d'appartenance ${ }^{11}$.

\section{LES MÉCANISMES DE LA PARTICIPATION}

Une des façons d'identifier les pratiques d'intervention et leur modalité d'inscription dans l'espace social consiste à distinguer des «degrés » de participation. Il me semble en effet important de chercher à qualifier ou à repérer les modulations des expériences de participation. Je distingue trois degrés. À un premier degré, que je qualifierai d'infrapolitique, l'individu n'intervient qu'en tant que consommateur (ou spectateur, ou auditeur, etc.). Il s'agit d'une forme plutôt passive de participation, celle qui est sollicitée par exemple par les produits standard de l'industrie culturelle. Comme le disait Hegel à propos de la société civile, il s'agit d'un champ de l'action consciente qui demeure «borné»: le processus en

11. Sur la question des réseaux internationaux et du cosmopolitisme en art, voir Guy Bellavance (dir.), 2000 et Jean Fisher (dir.), 1994. 
effet ne concerne que l'expérience d'un individu atomisé aux prises avec ses besoins et ses intérêts égoïstes. À un deuxième degré, quasi-politique celui-là, l'individu intervient en tant qu'usager ou bénéficiaire. Il n'est en fait qu'un agent dans une chaîne d'opérations, le détenteur de certains droits instrumentalisés au sein d'un appareil dispensateur de service, comme le voyageur dans le réseau de transports en commun ou devant un écran d'information. La marge d'action est réduite à une procédure convenue, une série de décisions et de gestes prévisibles. On pourrait aussi rapprocher ce genre de participation à des formes de représentation très ritualisées comme le Ballet comique de la reine à la cour de France, en 1581: on est bien dans la représentation du pouvoir, avec ses intrigues, mais certainement pas dans le politique! Dans une grande part de l'art public traditionnel, qui obéit d'abord à des fonctions commémoratives, décoratives ou didactiques, on tiendra compte de l'individu comme destinataire, plus ou moins réceptif, capable de partager un certain nombre de valeurs, de normes, de conventions qui définissent l'espace public. Le troisième degré désigne l'intervention de l'individu en tant que citoyen actif; c'est ici que le passage au politique devient possible. L'individu entre dans le processus de création non plus seulement en tant que consommateur ou usager, mais comme une personne qui se conçoit des appartenances, qui se raconte et qui dialogue, qui ainsi peut apparaître et agir en commun.

Sur ce plan, la participation de l'individu déborde la définition technique ou opératoire du processus et confine à une dynamique proprement politique. L'art se place en terrain instable parce qu'il s'ouvre à la différence des convictions et des perceptions, à la représentation du conflit, à la délibération.

Ce qui se passe en fait c'est que l'art s'ouvre toujours davantage à ce qui n'est pas lui. C'est donc dire que les artistes ne se contentent pas de revêtir les habits de l'intellectuel ou du philosophe pour s'investir d'une nouvelle mission sacrée. Les artistes sont véritablement engagés dans quelque chose, avec d'abord tous les gens qui se joignent au processus de l'œuvre, mais éventuellement aussi tous les autres qui en deviennent les médiateurs, les historiens, les critiques. Ils sont donc tout autant dans l'espace politique que dans le monde de l'art, et ils ne peuvent échapper aux aléas du politique: malentendu, détournement, mensonge et autres distorsions du langage, problèmes de légitimité, conflit d'intérêts, expressions contradictoires du bien commun et de la vie bonne, etc. Finalement, la participation du citoyen au processus de l'œuvre implique une réflexion sur les fondements mêmes du politique comme l'appartenance, la promesse, la sollicitude, l'échange, ce que signifie être et vivre ensemble.

Sur un plan très pratique ou stratégique, une question déterminante est de savoir quelle position occupent les artistes et leurs projets par rapport aux expériences de citoyenneté issues des milieux communautaires (groupes de lutte contre la pauvreté, l'exclusion, la violence, la marginalisation, les expériences de promotion ou de revitalisation communautaire, etc.), d'une part, et celles qui, d'autre part, figurent dans les projets officiels de redéfinition de la citoyenneté, au Québec ou ailleurs (politiques en matière d'immigration et de droits de la personne, le droit des peuples autochtones, la réforme du processus électoral et des institutions, etc.). Une autre question déterminante concerne la part de réflexion, d'invention ou d'expérimentation artistique qui entre dans le processus de créa- 
tion, par rapport à l'activisme social ou politique, par rapport aux préoccupations d'ordre strictement communautaire ${ }^{12}$.

\section{CONCLUSION : CRITIQUE, ASSOM PTION ET RÉGRESSION DU SPECTACULAIRE}

À partir des questions que soulève la définition même du genre - les interventions artistiques en milieu urbain -, il devient donc nécessaire de lancer quelques perspectives de réflexion sur la société globale et contribuer à la discussion sur le spectacle de la ville. Ce faisant, je souhaite montrer quelle sorte de contribution la sociologie de l'art peut apporter à la théorie de la société, sans perdre de vue les spécificités formelles et normatives des œuvres d'art, des objets singuliers qu'elle place au centre de son attention.

À cet égard, je crois utile d'articuler une théorie de l'œuvre d'art qui ne nous oblige pas à un choix exclusif entre les grandes traditions herméneutiques, sémiotiques et sociologiques, mais cherche plutôt à les croiser. Je ne le ferai pas ici. Mon argument est que les nouvelles formes d'intervention artistique en milieu urbain interrogent la structure même de l'espace public et l'inscription des personnes dans cet espace. En elles nous voyons s'affronter les intérêts divergents mais parfois convergents de la création, de l'expérimentation, de la diffusion artistique, d'une part, et du divertissement, du développement économique ou récréotouristique, de la gestion publique, d'autre part. L'interprétation sociologique doit alors comprendre comment l'autonomie spécifique des décideurs comme des artistes s'exerce dans un environnement qui connaît des contraintes elles aussi spécifiques (organisationnelles, sociales, politiques, esthétiques).

Ainsi que Bianchini le demandait, les politiques culturelles sont-elles condamnées à agiter des «masques de carnaval » pour camoufler les nouvelles inégalités urbaines, sontelles à jamais réquisitionnées comme « ciment social » pour intégrer les immigrants, favoriser l'insertion et la cohésion sociales? Des controverses importantes subsisteraient, liées aux débats sur la citoyenneté et sur la démocratie: lutte contre la réduction fonctionnaliste, la standardisation, l'érosion de l'urbanité, et pour une expérience de la vie urbaine publique plus diverse, plus riche. Ici comme ailleurs cependant, l'art n'est pas réductible à la spontanéité ou l'immédiateté revendiquée par les uns ou les autres, l'art ne doit pas se résumer à l'expression d'une identité ; il est l'enjeu de conflits idéologiques et esthétiques propres, de stratégies culturelles, le lieu d'une appropriation toujours problématique. Sur le plan philosophique, cette situation inédite dans l'histoire des villes et des civilisations impose de trouver un dialogue entre protection, revitalisation et création, entre les marques du passé, le devoir de transmission et de mémoire, et le devoir d'oubli, le décloisonnement des mondes possibles.

Les concepts de «nouveau lieu culturel» et de «nouvel espace public» ne s'inscrivent pas seulement dans la recherche d'un art hors institution, commune à une multitude de pratiques artistiques depuis au moins les années 1960. La Factory d'Andy Warhol gardait

12. Ces questions ont, entre autres, fait l'objet d'intéressantes discussions lors du colloque «Art actuel et espace public » organisé par le Centre d'information Artexte à l'automne 2004. Le volet artistique comportait des interventions de Christian Barré, Mathieu Beauséjour, James Carl et Minerva Cuevas. 
peut-être un côté exclusif; la Place des arts de Roussil et Vaillancourt en 1953, en revanche, était certainement animée par un idéal populaire. Ces nouveaux lieux auxquels les artistes cherchent à donner forme s'inscrivent maintenant dans une dialectique des identités qui s'étend bien au-delà du système de l'art et de ses contradictions internes.

À Montréal, dans la course pour occuper la scène urbaine, on observe en fait les mouvements les plus opposés: un important budget d'intégration des arts à l'architecture, divisé en quatre concours distincts, dans le cas du nouvel édifice de la Bibliothèque nationale du Québec (la Grande Bibliothèque); un design audacieux, des œuvres d'intégration et des aménagements paysagers pour la nouvelle section du Palais des congrès; la relocalisation de La joute qui devient une véritable attraction; la multiplication de gestes anonymes et furtifs, les prises de parole décentrées ou encore subversives, qui en appellent à une spécificité des pratiques artistiques interventionnistes.

L'argument que j'ai développé ici, sur la redéfinition de l'espace public à partir des expériences concrètes d'intervention artistique en milieu urbain, peut se rattacher de façon lointaine aux thèses défendues par Jürgen Habermas $(1978,1997)$, qui indiquait la possibilité d'un rôle actif propre à la société civile. La société civile est pensée par lui comme une instance de médiation entre les structures abstraites du système politique et les pratiques communicationnelles du monde vécu. Ici, cependant, les interventions artistiques n'ont pas nécessairement à attendre leur dramatisation et leur dissémination dans les médias de masse pour atteindre leur public et accéder à l' «agenda public», et elles n'ont pas obligatoirement à emprunter les voies de la délibération pragmatique pour former l'«opinion ». On pourrait par ailleurs les considérer comme le signe le plus éclatant de la disparition du politique ou de la fin du politique. Les interventions «micropolitiques » exprimeraient alors surtout le désenchantement des citoyens (Uzel, 2003). Si elles peuvent effectivement exprimer une désaffection à l'égard des institutions et des mécanismes du pouvoir, elles se présentent aussi comme des expériences concrètes de voies alternatives. Loin de se tenir en deçà du politique, elles chercheraient alors plutôt à s'articuler, non pas aux processus classiques de la délibération et de la formation de l'opinion, mais aux fondements pragmatiques, normatifs et expressifs du politique. Cette dernière hypothèse me semble caractériser le champ des interventions artistiques et correspondre aux mouvements contradictoires de redéfinition de la citoyenneté aujourd'hui.

La critique du spectaculaire, dans la mouvance de l'Internationale situationniste mais peut-être surtout avec les interventions micro-utopiques des années 1990, tente de mettre au jour les déterminations du système de l'art et de redonner à l'espace social (au sens arendtien de "monde mis en commun») la sorte de fondement ou d'initiative nécessaire à une expérience culturelle non réifiée. Cette mise au jour passe par la redéfinition de l'espace public, par delà les catégories léguées par la tradition moderne. Si la sociologie veut dire quelque chose de pertinent sur ce genre d'œuvre qui rompt avec l'esthétique de la représentation, voire avec toute forme d'objectalité, elle va devoir non seulement continuer à démasquer le jeu de l'art, mais comprendre ses nouveaux territoires et son hétéronomie complexe, comprendre comment les pratiques artistiques s'immiscent dans l'espace social. 


\section{RÉSUMÉ}

L'article attire l'attention sur un ensemble de pratiques artistiques qui relèvent de l'«intervention », de la «performance » et de l'«installation » et qui entendent déconstruire le spectacle des villes. Les interventions artistiques en milieu urbain remettent en question les catégories usuelles de l'art et du spectateur, mais aussi celles de l'action culturelle, de l'interaction et de la communauté. Partant de l'hypothèse générale que les «œuvres d'art» - qui ne sont pas nécessairement des « objets »-sont des configurations signifiantes, on examine les discours critiques et esthétiques qui concourent à la définition du genre, puis les éléments clés du champ de l'intervention artistique: la revitalisation urbaine, les politiques culturelles, les réseaux artistiques, les nouveaux espaces publics et les formes de participation. L'article vise à démontrer que les artistes qui pratiquent l'intervention urbaine doivent directement faire face à la difficulté de penser le nouvel espace public et de le concrétiser.

\section{ABSTRACT}

The paper studies a set of artistic practices stemming from "intervention," "performance" and "installation," and which aim to deconstruct the spectacle of cities. Artistic interventions in an urban setting defy the usual categories of art and the spectator, but also those of cultural action, interaction and the community. Starting with the general hypothesis that "works of art"-which are not necessarily "objects"-are significant configurations, a study is made of the critical and aesthetic discourses which contribute to the definition of the genre, and secondly of the key elements in the field of artistic intervention: urban renewal, cultural policies, artistic networks, new public spaces and forms of participation. The paper aims to show that the artists who work in an urban setting must face head-on the problem of defining the new public space and giving it concrete form.

\section{RESUMEN}

El artículo llama la atención sobre un conjunto de prácticas artísticas que son de la competencia de "la intervención", de la "prestación" y de la "instalación", y que pretenden desconstruir el espectáculo de las ciudades. Las intervenciones artísticas en medio urbano cuestionan las categorías usuales del arte y el espectador, y también las de la acción cultural, la interacción y la comunidad. A partir de la hipótesis general de que las "obras de arte"-que no son necesariamente "objetos"-son configuraciones significativas, se examinan los discursos críticos y estéticos que concurren en la definición del género, y los elementos claves del campo de la intervención artística: la revitalización urbana, las políticas culturales, las redes artísticas, los nuevos espacios públicos y las formas de participación. El artículo tiene por objeto demostrar que los artistas que practican la intervención urbana se enfrentan directamente a la dificultad de pensar el nuevo espacio público y de concretarlo.

\section{BIBLIOGRAPHIE}

Alexander, J. et Pн. Smith (1998), «Sociologie culturelle ou sociologie de la culture? Un programme fort pour donner à la sociologie son second souffle», Sociologie et sociétés, vol. XXX, n 1, p. 107-116.

Araeen, R. (2000), «A New Beginning: Beyond Postcolonial Cultural Theory and Identity Politics», Third Text, $\mathrm{n}^{\circ}$ 50, p. 3-20.

Ardenne, P. (2002), Un art contextuel: création artistique en milieu urbain, en situation d'intervention, de participation, Paris, Flammarion. 
Ardenne, P. (2001), «L'art public: ambiguïté et crise de l'impact», dans Patrice Loubier et Anne-Marie Ninacs (dir.), Les commensaux: quand l'art se fait circonstances, Montréal, Skol, p. 35-44.

Alain, D. et Doyon/Demers, S. Pelletier, R. Richard (1999), Bulletin des Instants ruraux, Granby, $3^{\mathrm{e}}$ Impérial, Centre d'essai en arts visuels.

Alain, D. et Ronald et Richard, J. K. Grande, S. Pelletier, Y. Gendreau (2000), Instants ruraux 19981999, Granby, $3^{\mathrm{e}}$ Impérial, Centre d'essai en arts visuels.

Alain, D. et Ph. Côté, S. Pelletier, P. Loubier, Ronald et Richard (2002), Supra rural 1999-2000, Granby, $3^{\mathrm{e}}$ Impérial, Centre d'essai en arts visuels.

Augoyard, J.-Fr. (2000), «L'action artistique dans l'espace urbain», dans Jean Métral (dir.), Cultures en ville, ou de l'art et du citadin, La Tour D'Aigues, Éditions de l'Aube.

Babin, S. (2001), «Pratiquer la ville», Esse, nº 42, printemps-été, p. 6-21.

BAudöCK, R. (1992), Immigration and the Boundaries of Citizenship, Warwick, Centre for Research in Ethnic relations, «Monographs in Ethnic relations», $\mathrm{n}^{\circ} 4$.

Beaudet, P. (2003), «Considérations sur le déplacement de La Joute», Espace, n 64, été, p. 21-23.

BeCKer, H. S. (1983), «Mondes de l'art et types sociaux », Sociologie du travail, n ${ }^{\circ}$, p. 404-417.

Becker, H. S. (1982), Art Worlds, Berkeley, University of California Press.

Bellavance, G. (dir.) (2000), Monde et réseaux de l'art: diffusion, migration et cosmopolitisme en art contemporain, Montréal, Liber.

Bellavance, G., L. Bernier et B. Laplante (2001), Les conditions de pratique des artistes en arts visuels: Rapport d'enquête, phase 1, Montréal, Regroupement des artistes en arts visuels (RAAV).

Bellavance, G. en coll. avec L. Santerre et M. Boivin (dir.) (2000), Démocratisation de la culture ou démocratie culturelle?, Québec, Les Presses de l'Université Laval.

BHARUCHA, R. (1999), «Interculturalism and its Discriminations: Shifting the Agendas of the National, the Multicultural and the Global», Third Text, n 46, p. 3-23.

Bianchini, Fr. et M. Parkinson (dir.) (1993), Cultural policy and urban regeneration: the West European experience, Manchester, Manchester University Press.

Blau, J. R. (1989), «High Culture as Mass Culture», dans Arnold W. Foster et Judith R. Blau (dir.), Art and Society: Readings in the Sociology of the Arts, New York, SUNY Press, p. 429-439.

Boisvert, Y., J. Hamel, M. Molgat, avec la collaboration de B. Ellefsen (dir.) (2000), Vivre la citoyenneté: identité, appartenance et participation, Montréal, Liber.

Bourdieu, P. (1979), La distinction: critique sociale du jugement, Paris, Minuit.

Bourdieu, P. (1992), Les règles de l'art: genèse et structure du champ littéraire, Paris, Seuil.

Bourque, G., J. duchastel, et É. Pineault (1999), «L'incorporation de la citoyenneté », Sociologie et sociétés: Citoyenneté et identité sociale, vol. XXXI, nº 2, p. 41-64.

Bourriaud, N. (1998), Esthétique relationnelle, Dijon, Les Presses du Réel.

Chatterjee, P. (1998), «Beyond the Nation? Or Within?», Social Text, vol. 16, $\mathrm{n}^{\circ} 3$.

Côté, J.-Fr. (1998), «La société de communication à la lumière de la sociologie de la culture: idéologie et transmission de sens", Sociologie et sociétés, vol. XXX, nº 1, p. 117-132.

Couture, Fr. (2003), «Expositions collectives et montréalisation de l'art contemporain ", dans Francine Couture (dir.), Exposer l’art contemporain du Québec, Montréal, Centre de diffusion 3D, p. 55-97.

Couture, Fr. (1999), «Identités d'artiste», dans Marie-Charlotte De Koninck et Pierre Landry (dir.), Déclics: art et société, le Québec des années 1960 et 1970, Québec et Montréal, Musée de la civilisation, Musée d'art contemporain de Montréal et Fides, p. 51-83.

Crane, D. (1986), « Avant-garde Art and Social Change : The New York Art World and the Transformation of the Reward System, 1940-1980 ", dans Raymonde Moulin (dir.), Sociologie de l'art, Paris, La Documentation française, p. 69-82.

Crane, D. (1987), The Transformation of the Avant-Garde: The New York Art World, 1940-1985, Chicago et Londres, University of Chicago Press.

Crane, D. (dir.) (1994), The Sociology of Culture: Emerging Theoretical Perspectives, Oxford et Cambridge, Blackwell. 
D’Angelo, M., E. Freidberg et Ph. Urfalino, avec la collaboration de M.-A. Mazoyer (1989), Les politiques culturelles des villes et leurs administrateurs, Paris, La Documentation française.

De Koninck, M.-Сh. et P. LAndry (dir.) (1999), Déclics : art et société, le Québec des années 1960 et 1970, Québec et Montréal, Musée de la civilisation, Musée d'art contemporain de Montréal et Fides.

DiMaggio, P. (1991), «Social Structure, Institutions and Cultural Goods: The Case of the United States», dans Pierre Bourdieu et J. Coleman (dir.), Social Theory for a Changing Society, New York, R. Sage Foundation, p. 133-155.

DiMaggio, P. (1987), «Classification in Art », American Sociological Review, n ${ }^{\circ}$ 2, p. 440-455.

DoucEt, D. (2003), «Le déplacement de la sculpture-fontaine La Joute de Riopelle, un débat national en art public», Espace, nº 64, été, p. 24-27.

Doyon/Demers (2003), «Citoyen "volontaire" », Esse, n 48, p. 6-11.

Dufrasne, M. et J.-Fr. Prost (2000), «Convivialités électives. Entretien », Inter, nº 77, p. 33-37.

Elbaz, M. et D. Helly (dir.) (2000), Mondialisation, citoyenneté et multiculturalisme, Québec, les Presses de l'Université Laval.

Featherstone, M. (1991), Consummer Culture and Postmodernism, Londres, Sage Publications.

Fisher, H. (1977), Théorie de l'art sociologique, Paris, Casterman.

Fisher, J. (dir.) (1994), Global Visions: Towards a New Internationalism in the Visual Arts, Londres, Kala Press et The Institute of International Visual Arts.

Fortin, A. (2002), «L'art et la place publique», dans Traité de la culture, sous la dir. de Denise Lemieux, avec la coll. de Gilles Bibeau, Michelle Comeau, François-Marc Gagnon, Fernand Harvey, Marc-André Lessard et Gilles MArcotTe, Québec, les Éditions de l'IQRC, chap. 31, p. 595-606.

Fraser, M. et M.-J. Lafortune (dir.) (2001), Gestes d'artistes, Montréal, Optica.

Fraser, M., D. Gougeon et M. Perrault (dir.) (1999), Sur l'expérience de la ville: interventions en milieu urbain, Montréal, Optica.

Freidberg, E. et Ph. Urfalino (1984), Le jeu du catalogue: les contraintes de l'action cuturelle dans les villes, Paris, La Documentation française.

Freidson, E. (1986), «Les professions artistiques comme défi à l’analyse sociologique », Revue française de sociologie, XXVII, p. 431-443.

Friches industrielles, lieux culturels (1994), actes du colloque, Strasbourg, La Laiterie, Centre européen de la jeune création.

Grafmeyer, Y. (1999), «La coexistence en milieu urbain : échanges, conflits, transaction », Recherches sociologiques: sociologie de la ville, $\mathrm{n}^{\circ}$ 1, p. 157-176.

HAYs, S. (2000), «Constructing the Centrality of Culture, and Deconstructing Sociology? », Contemporary Sociology, vol. 29, n $\mathrm{n}^{\circ}$ 4, p. 594-602.

Habermas, J. (1978), L'espace public: archéologie de la publicité comme dimension constitutive de la société bourgeoise, trad. Marc B. Launay, Paris, Payot.

Habermas, J. (1997), «Le rôle de la société civile et de l'espace public politique», Droit et démocratie: entre faits et normes, trad. Rainer Rochlitz et Christian Bouchindhomme, Paris, Gallimard, chap. XVIII.

Harvey, D. (1990), The Condition of Postmodernity: An Inquiry into the Origins of Cultural Change, Oxford et Cambridge, Blackwell.

Heinich, N. (1992), « La partie de main chaude de l'art contemporain », dans Jean-Olivier MAJAstre et Alain Pessin (dir.), Art et contemporanéité, $\mathrm{r}^{\text {re }}$ Rencontre internationale de sociologie de l'art de Grenoble, Bruxelles, la Lettre volée, p. 81-110.

Honig, B. (1998), «Immigrant America? How Foreignness Solves Democracy's Problems», Social Text, vol. $16, \mathrm{n}^{\circ} 3$.

Huelsenbeck, R. (1974), Memoirs of a Dada Drummer, sous la dir. de Hans J. Kleinschmidt, trad. Joachim Neugroschel, New York, Viking Press.

JАСОВ, M. J. (dir.) (1995), Culture in Action: A Public Art Program of Sculpture Chicago, Seattle, Bay Press. JANOver, L. (1989), La révolution surréaliste, Paris, Plon.

Jeudy, H.-P. (1999), «L'art et l'espace public», Les usages sociaux de l'art, Paris, Circé, chap. 3, p. 105-130. 
KING, A. D. (dir.) (1997), Culture, Globalization and the World-System: Contemporary Conditions for the Representation of Identity, Minneapolis, University of Minnesota Press.

Kyмlicka, W. (1995), Multicultural Citizenship: A Liberal Theory of Minority Rights, Oxford, Clarendon Press.

LaCY, S. (dir.) (1995), Mapping the Terrain: New Genre Public Art, Seattle, Bay Press.

Lamarche, L. (1999), Textes furtifs: autour de la sculpture, 1978-1999, Montréal, Centre de diffusion 3D.

Lamont, M. (2000), «Meaning-Making in Cultural Sociology: Broadening Our Agenda », Contemporary Sociology, vol. 29, nº 4, p. 602-607.

Latouche, D. (2003), «La ville et ses diversités créatrices», dans Daniel Latouche (dir.), Voulez-vous manger avec moi? Pratiques interculturelles en France et au Québec, Montréal, Fides, chap. 11, p. 123-142.

LESSARD, M. (1997), " De l'usine à la création artistique: usages éphémères ou usages permanents? », dans Les nouveaux lieux culturels, actes du colloque, ix ${ }^{e}$ Entretiens du Centre Jacques-Cartier, Montréal, p. 63-70.

Lévesque, L. (1999), «Montréal, l'informe urbanité des terrains vagues. Pour une gestion créatrice du mobilier urbain ", Les Annales de la recherche urbaine: paysages en villes, $\mathrm{n}^{\circ}$ 85, p. 47-57.

Loubier, P. (2002), «Un art à fleur de réel: considérations sur l'action furtive», Inter, nº 81, p. 12-17.

Loubier, P. et A.-M. Ninacs (dir.) (2001), Les commensaux: quand l'art se fait circonstances, Montréal, Skol. Luhmann, N. (2000), Art as a Social System, trad. Eva M. Knodt, Stanford, Stanford University Press.

Menger, P.-M. (1999), «Artistic Labor Markets and Careers », Annual Reviews of Sociology, n 25, p. 541-574.

Menger, P.-M. (1989), «Rationalité et incertitude de la vie d'artiste », L'Année sociologique, vol. 39, p. 111-151.

Métral, J. (dir.) (2000), Cultures en ville, ou de l'art et du citadin, La Tour D’Aigues, Éditions de l'Aube.

Michaud, Y. (1997), La crise de l'art contemporain: utopie, démocratie et comédie, Paris, PUF.

Ministère de L'aménagement du terRitoire et des ReConversions (1991), La réhabilitation des friches industrielles, Paris, La Documentation française.

Miller, T. (1998), «Exchange-Value Citizenship?», Social Text, vol. 16, nº 3 .

Nielsen, G. M., Yon Hsu et Louis Jасов (2002), « Public Culture and the Dialogics of Democracy: Reading the Montréal and Toronto Amalgamation Debates ", Canadian Journal of Urban Research: Space, Place and the Culture of Cities, vol. XI, $\mathrm{n}^{\circ} 1, \mathrm{p} .111-139$.

Pelletier, S. (2001), «Pratiques urbaines ou art universel issu d'un contexte urbain?», Esse, no 42, printemps-été, p. 22-27.

RAFFin, F. (2000), «Du nomadisme urbain aux territoires culturels : la mise en culture des friches industrielles à Poitiers, Genève et Berlin ", dans Jean Métral (dir.), Cultures en ville, ou de l’art et du citadin, La Tour D'Aigues, Éditions de l'Aube, p. 51-68.

Regroupement des centres d'artistes autogérés du Québec (RCAAQ) (2003), «Centres d'artistes autogérés et Quartier des spectacles », document rédigé en vue des audiences publiques de la Commission d'urbanisme de la Ville de Montréal.

Richard, Alain-Martin (2001), «L'art action au Québec: corps privé et corps public», dans Richard MARTeL, Pierre Restany, Jean-Jacques Lebel et al., Art Action 1958-1998, Québec, Éditions Intervention.

Rioux, J.-P. et J.-Fr. Sirinelli (dir.) (1997), Pour une histoire culturelle, Paris, Seuil.

Rodriguez, V. (1995), «Un cas de gentrification artistique dans le milieu montréalais », examen de synthèse, Département de sociologie, Université de Montréal.

SAssen, S. (1996), Losing Control? Sovereignty in an Age of Globalization, New York, Columbia University Press.

Simpson, C. R. (1981), SoHo: The Artist in the City, Chicago et Londres, University of Chicago Press.

Sioui Durand, G. (2002), "Quand les attitudes d'art deviennent stratégies ", Inter, nº 81, p. 20-23.

Sioui Durand, G. (1997), L'art comme alternative: réseaux et pratiques d'art parallèle au Québec, 1976-1996, Québec, les Éditions Intervention.

Smiers, J. (1998), État des lieux de la création en Europe : le tissu culturel déchiré, Paris et Montréal, L'Harmat$\tan$.

Thuriot, F. (1999), Culture et territoires : les voies de la coopération, Paris et Montréal, l'Harmattan.

Uzel, J.-Рн. (2003), «L'art micropolitique est-il politique?, Esse, nº 48, p. 12-15. 
Ville de MontréAl (2003a), «Cadre, principes directeurs et énoncé de politique culturelle», rapport du Groupe-conseil présidé par Raymond Bachand.

Ville de Montréal (2003b), "Le rôle de la Ville de Montréal en matière d'art public », Service du développement culturel, document déposé à la Commission permanente du conseil sur les arts, la culture et le patrimoine.

VILLE DE MonTRÉAL (2003c), «Culture et arrondissement », document préparé pour la consultation publique de la Commission permanente du conseil sur les arts, la culture et le patrimoine.

Ville De Montréal (1989), «L'art public à Montréal : Énoncé de politique et de programmes. Plan triennal d'action ", CIDEC.

Zızeк, S. (1997), «Multiculturalism, The Cultural Logic of Multinational Capitalism», New-Left Review, $\mathrm{n}^{\circ} 225$, p. 28-51.

Zolberg, V. (1990), Constructing a Sociology of the Arts, Cambridge, Cambridge University Press.

Zukin, S. (1982), Loft Living: Culture and Capitalism in Urban Change, Baltimore, Johns Hopkins University Press. 